\title{
T cells expressing the lupus susceptibility allele Pbx1d enhance autoimmunity and atherosclerosis in dyslipidemic mice
}

\author{
Wei Li, ${ }^{1}$ Ahmed S. Elshikha, ${ }^{1,2}$ Caleb Cornaby, ${ }^{1}$ Xiangyu Teng, ${ }^{1}$ Georges Abboud, ${ }^{1}$ \\ Josephine Brown, ${ }^{1}$ Xueyang Zou, ${ }^{1,3}$ Leilani Zeumer-Spataro, ${ }^{1}$ Brian Robusto, ${ }^{1}$ Seung-Chul Choi, ${ }^{1}$ \\ Kristianna Fredenburg, ${ }^{1}$ Amy Major, ${ }^{4,5}$ and Laurence Morel $^{1}$ \\ 'Department of Pathology, Immunology and Laboratory Medicine, University of Florida, Gainesville, Florida, USA. \\ 2Department of Pharmaceutics, Zagazig University, Zagazig, Sharkia, Egypt. ${ }^{3}$ Department of Immunology, College of \\ Basic Medical Sciences, Jilin University, Changchun, China. ${ }^{4}$ Department of Medicine, Division of Rheumatology and \\ Immunology, Vanderbilt University Medical Center, Nashville, Tennessee, USA. 5U.S. Department of Veterans Affairs, \\ Tennessee Valley Healthcare System, Nashville, Tennessee, USA.
}

Patients with systemic lupus erythematosus (SLE) present a high incidence of atherosclerosis, which contributes significantly to morbidity and mortality in this autoimmune disease. An impaired balance between regulatory (Treg) and follicular helper (Tfh) $C D 4^{+} T$ cells is shared by both diseases. However, whether there are common mechanisms of $C D 4^{+} \mathrm{T}$ cell dysregulation between SLE and atherosclerosis remains unclear. Pre-B cell leukemia transcription factor 1 isoform $\mathrm{d}(P b x 1 d)$ is a lupus susceptibility gene that regulates Tfh cell expansion and Treg cell homeostasis. Here, we investigated the role of T cells overexpressing Pbx1d in low-density lipoprotein receptor-deficient $\left(L d l r^{--}\right)$mice fed with a high-fat diet, an experimental model for atherosclerosis. Pbx1d-transgenic T cells exacerbated some phenotypes of atherosclerosis, which were associated with higher autoantibody production, increased Tfh cell frequency, and impaired Treg cell regulation, in Ldlr $^{\text {/- }}$ mice as compared with control T cells. In addition, we showed that dyslipidemia and Pbx1dtransgenic expression independently impaired the differentiation and function of Treg cells in vitro, suggesting a gene/environment additive effect. Thus, our results suggest that the combination of Pbx1d expression in T cells and dyslipidemia exacerbates both atherosclerosis and autoimmunity, at least in part through a dysregulation of Treg cell homeostasis.

Conflict of interest: The authors have declared that no conflict of interest exists.

Copyright: () 2020, American Society for Clinical Investigation.

Submitted: March 18, 2020

Accepted: May 6, 2020

Published: June 4, 2020

Reference information: /CI Insight. 2020;5(11):e138274.

https://doi.org/10.1172/jici.

insight.138274.

\section{Introduction}

As the underlying cause of heart attacks and strokes, atherosclerosis is one of the leading causes of death worldwide. Mechanistically, it is a lipid-driven chronic disease in which leukocyte accumulation in the vessel wall actively participates in the disease process (1). A higher incidence of atherosclerosis has been reported among patients with systemic lupus erythematosus (SLE) (2), with a large cross-sectional study reporting a 1.45 odds ratio of hospitalized SLE patients to present with atherosclerosis (3). A potential mechanism linking SLE and atherosclerosis is that both diseases are mediated by activated T cells $(4,5)$. Using mouse models, it has been recently reported that dyslipidemia exacerbates SLE pathogenesis with enhanced follicular helper $\mathrm{T}$ ( $\mathrm{Tfh}$ ) cell responses (6), while transferring $\mathrm{CD} 4^{+} \mathrm{T}$ cells from lupus-prone mice into dyslipidemic mice accelerates the development of atherosclerosis $(7,8)$. These results suggest that $\mathrm{CD}^{+} \mathrm{T}$ cells represent a functional link in a reciprocal enhancement between SLE and atherosclerosis. Furthermore, an imbalance between impaired regulatory $\mathrm{CD} 4^{+} \mathrm{Foxp} 3^{+} \mathrm{T}$ (Treg) cells and activated effector $\mathrm{CD}^{+} \mathrm{T}$ (Teff) cells has been shown to have a causative effect in atherosclerotic mouse models (9-11). A Treg/Teff imbalance is also found in patients with SLE (4). Thus, there is a growing interest in identifying common mechanisms by which $\mathrm{CD}^{+} \mathrm{T}$ cells induce pathogenesis in both SLE and atherosclerosis.

We have identified 3 major lupus susceptibility loci in the NZM2410 mouse model, which are necessary and sufficient to induce lupus nephritis on a non-autoimmune B6 background in the B6.Sle1.Sle2.Sle3 triple congenic (TC) strain (12). Transferring BM or $\mathrm{CD}^{+} \mathrm{T}$ cells from $\mathrm{TC}$ mice accelerated atherosclerosis in the aortic sinus of low-density lipoprotein receptor-deficient $(L d l r-\mathrm{KO})$ dyslipidemic mice, characterized 
by increased T cell infiltration in lesions. Enhanced atherosclerosis was independent of serum cholesterol or triglycerides but was associated with an increase of spleen cell number, serum creatinine, serum urea, and anti-dsDNA antibody production $(7,8,13)$, indicating that dysregulated immunity was mechanistically involved. Furthermore, this suggested that the genetic loci, sufficient to induce lupus pathogenesis, also accelerate atherosclerosis through $\mathrm{CD}^{+}$effector $\mathrm{T}$ cell-mediated mechanisms. In the Ldlr-KO chimera, Treg cells from TC origin produced more IL-17 than Treg cells from B6 origin, suggesting that dysfunctional Treg cells may promote atherosclerotic lesions in those mice (8). Interestingly, the lupus susceptibility locus Sle3, one of 2 associated with T cell hyperactivation in the TC strain (14), was not sufficient to accelerate atherosclerosis (15), suggesting that Sle1 may accelerate atherosclerosis in lupus because Sle2 regulates autoreactive B cell phenotypes (16).

The Sle1 locus is necessary for the development of systemic autoimmunity in the NZM2410 model $(12,17)$, and it intrinsically regulates the function of $\mathrm{CD}^{+} \mathrm{T}$ cells (18). We show here that Sle1 accounts for a large part of the atherosclerosis-accelerating effect of the TC hematopoietic cells in the Ldlr-KO chimera model, which corroborates the major role CD4 ${ }^{+} \mathrm{T}$ cells play in this process. Sle1 contains at least 3 independent loci: Sle1a1, Sle1b, and Sle1c (19). Sle1a1 affects primarily $\mathrm{CD}^{+} \mathrm{T}$ cells by promoting the generation of autoreactive $\mathrm{T}$ cells (20) and impairing Treg functions $(20,21)$. Pre-B cell leukemia transcription factor $1(P b x I)$, the only gene located in Sle1a1, encodes for a transcription factor regulating multiple cellular processes through 7 splice isoforms (22). The Pbxld isoform lacks the DNA and the HOX binding domains, resulting in dominant negative functions (23), and its expression is increased in the $\mathrm{CD}^{+} \mathrm{T}$ cells from B6.Sle1a1 mice and SLE patients as compared with healthy controls (24). Transgenic (Tg) expression of Pbxld in $\mathrm{CD}^{+} \mathrm{T}$ cells reproduced the phenotypes of lupus $\mathrm{CD}^{+} \mathrm{T}$ cells, including decreased Treg cell homeostasis and Tfh cell expansion (25). Because these $\mathrm{T}$ cell populations have been implicated in atherosclerosis, we hypothesized that the overexpression of $\mathrm{Pbx} 1 \mathrm{~d}$ in $\mathrm{T}$ cells would exacerbate the development of atherosclerosis by impairing Treg cells, either in number or in function, and by expanding Teff cells in atherogenic, dyslipidemic conditions. Here, we show that Pbx1d-Tg CD4 ${ }^{+} \mathrm{T}$ cells enhanced atherosclerotic phenotypes associated with greater severity in $L d l r-\mathrm{KO}$ mice fed with a high-fat Western diet (WD). The overexpression of Pbx1d in T cells of dyslipidemic $L d l r-\mathrm{KO}$ mice also enhanced their autoimmune phenotypes, including autoantibody production, increased Tfh cell frequency, and impaired Treg cell regulation. In vitro studies suggest that excess cholesterol impaired the suppressive function of Treg cells, which, combined with the alteration in Treg homeostasis driven by Pbx1d, may be a mechanism by which this lupus susceptibility allele enhances both atherosclerosis and autoimmunity in dyslipidemic conditions.

\section{Results}

The atherosclerotic effect of immune cells from the TC lupus-prone mouse partially maps to Sle1. Because CD4 ${ }^{+} \mathrm{T}$ cells from TC mice are sufficient to accelerate atherosclerosis in dyslipidemic mice (8), we evaluated the B6.Sle1 and B6.Sle3 single congenic mice in this model (Figure 1A). Autoreactive CD4 ${ }^{+} \mathrm{T}$ cells are activated in these 2 strains, either directly for Sle1 (18) or indirectly for Sle3 (26), while Sle2, which regulates only B cells (27), was not evaluated in this experiment. Previous studies have demonstrated that hematopoietic transfer of cells expressing Sle3 is not sufficient to increase atherosclerosis in $L d l r-\mathrm{KO}$ mice. Previous work showed that all atherosclerotic phenotypes, including lesion size and severity in the aorta as well as the number of infiltrating $\mathrm{CD}^{+} \mathrm{T}$ cells and macrophages, were similar between Sle3 chimeras with B6 controls (15). The Sle3 chimeras are therefore functional controls with activated T cells that do not induce atherosclerosis. The Sle1 and TC LdlrKO chimeras showed similarly enlarged plaques measured by oil red O (ORO) stain (Figure 1, B and C), as compared with the Sle3 chimeras. Increased lesions were not due to increased levels of circulating cholesterol (Figure 1D) or triglycerides (data not shown). Accordingly, both Sle1 and TC Ldlr-KO chimeras presented increased infiltrates of $\mathrm{CD}^{+} \mathrm{T}$ cells in the lesions (Figure 1E). The Sle1 bone marrow-derived (BM-derived) cells were, however, not sufficient to induce autoantibodies against dsDNA or oxidized LDL (oxLDL) (Figure 1, F and G) to the same level as TC BM-derived cells. These results indicate that immune cells expressing Sle1 promote atherosclerosis at least in part through $\mathrm{CD}^{+} \mathrm{T}$ cells, independent of autoantibody and overt autoimmunity as observed in TC recipients.

Pbxld expression in $T$ cells enhanced atherosclerosis in Ldlr-KO mice fed with WD. We next investigated whether expression of the lupus susceptibility allele Pbxld, a major contributor to the Sle1 phenotypes in $\mathrm{CD}^{+} \mathrm{T}$ cells, could affect the development of atherosclerosis. We reconstituted lethally irradiated $L d l r_{-}$ $\mathrm{KO}$ mice with BM from Pbx1d-Tg.Foxp3 ${ }^{\text {YFPCre }}$ mice or B6.Foxp3 ${ }^{\text {YFPCre }}$ mice, which differ only by the overexpression of Pbxld in T cells (25). Six weeks after BM transfer, the chimeric mice were fed with WD to 
A

Recipients: LDLr-1
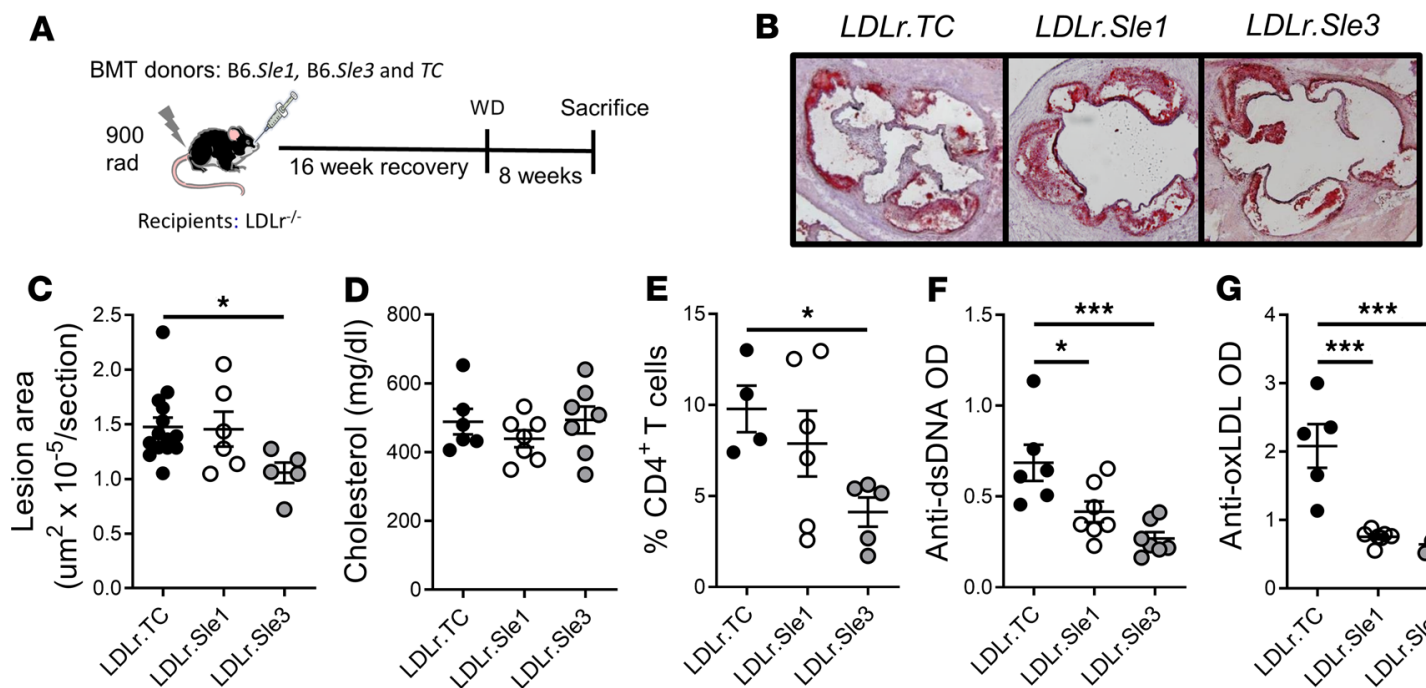

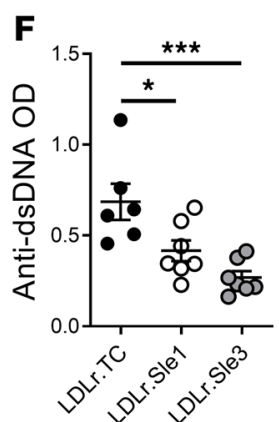

Figure 1. Sle1 partially accounts for the atherogenic effect of TC immune cells. (A) Experimental design. (B) Representative images of atherosclerotic lesions in the aortic root for each of the 3 groups and (C) corresponding morphometric analysis. (D) Terminal serum cholesterol. (E) Morphometric analysis of CD4+ $\mathrm{T}$ cell infiltrates in the aortic root. Terminal serum anti-dsDNA IgG (F) and oxLDL IgG (G). Means \pm SEM compared with 1-way ANOVA with Tukey's multiple-comparisons tests. ${ }^{*} P<0.05,{ }^{* *} P<0.001$. Each symbol represents 1 mouse.

induce hyperlipidemia or normal diet (ND) for another 8 weeks. As expected, WD increased body weight in both strains, but the Pbx1d chimeric mice gained more weight than the B6 chimeric mice (Figure 2A). WD increased serum triglycerides, total cholesterol, and LDL, as compared with ND, but as for the TC and single congenic chimeras (Figure 1D), there was no difference between strains (Figure 2, B-D). Also, as expected, WD induced the development of plaque in the aortic root that was larger than that of mice fed with ND (Figure 2E). There was no difference in the size of the plaque between the Pbx1d and B6 chimera (Supplemental Figure 1, A and B; supplemental material available online with this article; https:// doi.org/10.1172/jci.insight.138274DS1). Scoring on a semiquantitative scale the aortic valve histiocytic inflammation, perivalvular chronic inflammation or fibrosis, as well as the number of apoptotic cells did not reveal a difference between the Pbx1d and B6 chimera on WD (Supplemental Figure 1, C and D). However, a morphometric analysis revealed a thickening of the aortic tunica media of the Pbx1d chimera in the WD group, which showed higher values than either B6 chimera in the WD group, or Pbx1d chimera in the ND group (Figure 2, E and F). Dyslipidemia expands the tunica intima and media with an alteration of the elastic fibers, as well as an infiltration of Teff cells (28), which is most likely enhanced by Pbx1d-expressing T cells. In addition, the plaque in Pbx1d chimeric mice in the WD group showed larger necrotic cores (Figure 2G and Supplemental Figure 1E), corresponding to an accumulation of necrotic macrophages as a consequence of high levels of inflammation (29). Neither B6 nor Pbx1d chimeras in the ND group presented lesions that were advanced enough to develop a necrotic core (data not shown).

The surrounding myocardium of Pbx1d chimeras contained a higher number of infiltrating cells, and a similar trend was observed in the aorta (Figure $2 \mathrm{H}$ ). Among these cells, WD expanded monocytes to a similar extent in $\mathrm{B} 6$ and $\mathrm{Pbx} 1 \mathrm{~d}$ chimeras. However, $\mathrm{MHC}-\mathrm{II}^{\mathrm{lo}}$ macrophages were uniquely expanded in the heart of Pbx1d chimeras in the WD group (Figure 2I). These cells correspond to resident cardiac macrophages that have the highest capacity for efferocytosis and antigen processing (30). No difference was observed for circulating inflammatory macrophages (data not shown). The number of $\mathrm{CD} 4^{+} \mathrm{T}$ cells infiltrating the heart was the highest in Pbxld chimeras in the WD group, and a similar trend was observed in the aorta (Figure 2J). Furthermore, the frequency of CD4 ${ }^{+} \mathrm{T}$ cells expressing high levels of CD25, the majority of which are likely to be Treg cells, was reduced in the heart and aorta of Pbx1d chimeric mice regardless of the diet (Figure $2 \mathrm{~K}$ ). The number of $\mathrm{CD} 4{ }^{+} \mathrm{CD} 25^{+}$cells was similar among the groups (Supplemental Figure $1 \mathrm{~J}$ ), but a reduced CD25 expression was observed on the $\mathrm{CD}^{+} \mathrm{T}$ cells in the aorta of dyslipidemic Pbx1d chimeras (Figure 2L), a phenotype that has been reported in Treg cells in lupus and associated with their dysfunction (31). These results suggest that Pbx1d expression in $\mathrm{T}$ cells worsened atherosclerosis in $L d l r-\mathrm{KO}$ mice fed with WD. This was associated with an increased cardiovascular infiltrate and an expansion of resident macrophages and proinflammatory $\mathrm{CD} 4^{+} \mathrm{T}$ cells. 

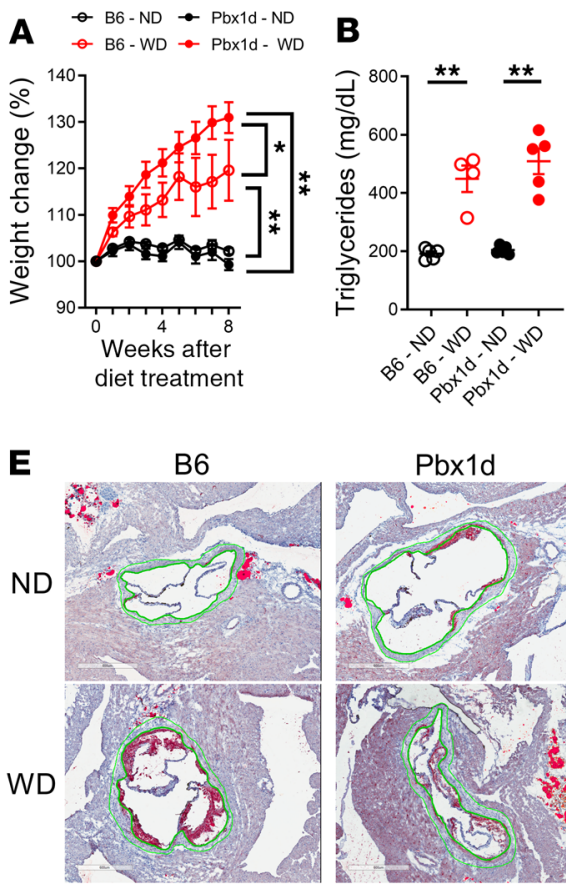

Pbx1d

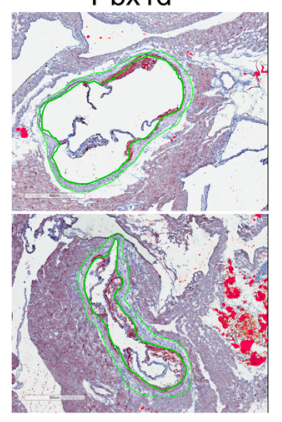

D
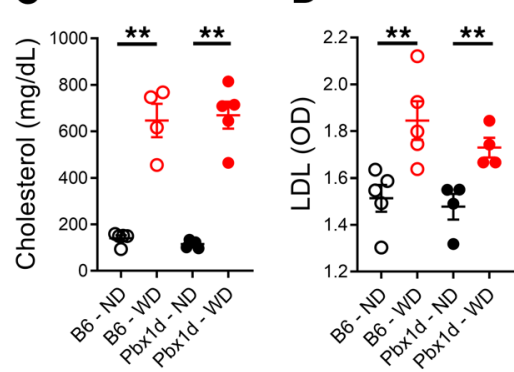

F Tunica media

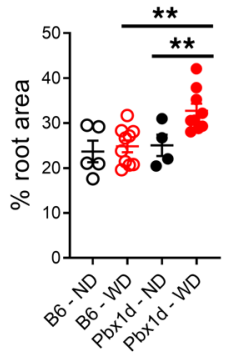

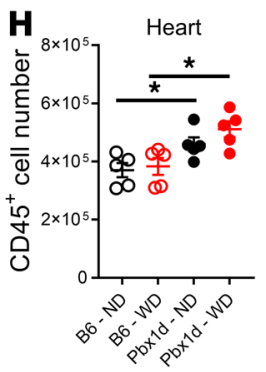
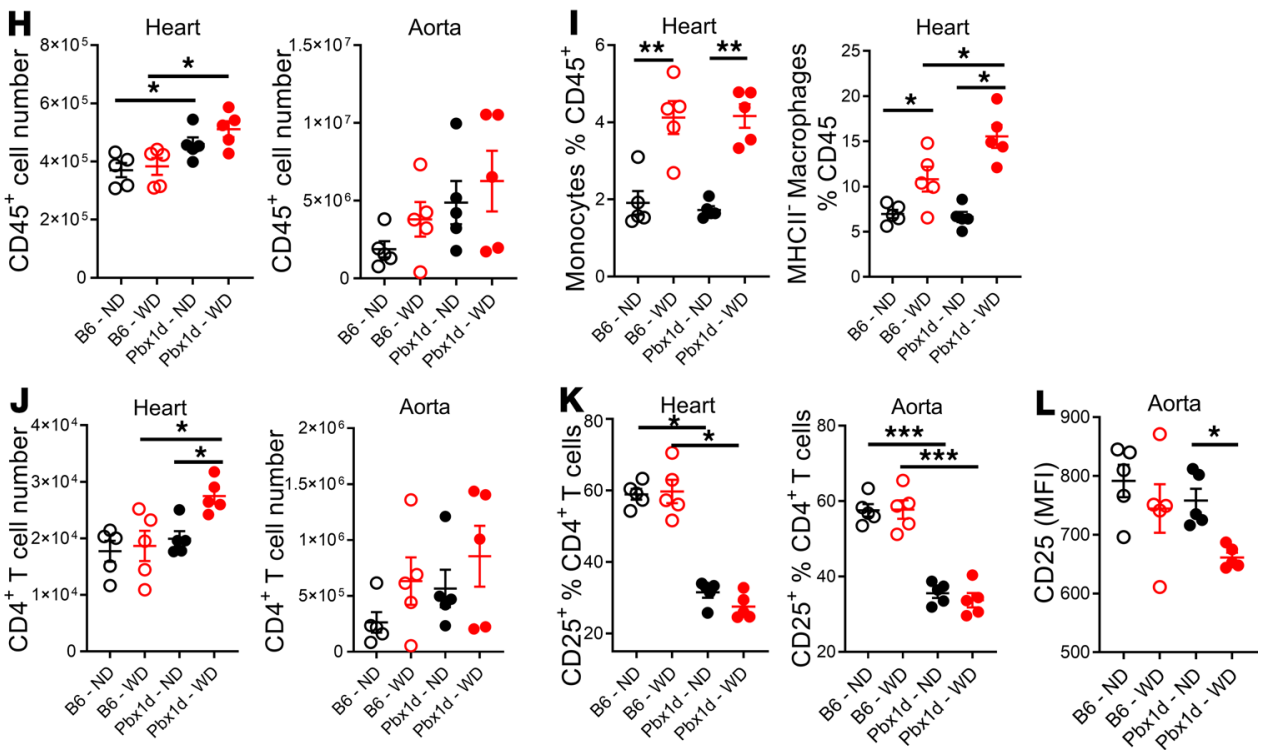

Figure 2. Pbx1d overexpression in CD4+ $\mathrm{T}$ cells accelerates atherosclerosis in Ldlr-KO mice fed with WD. (A) Body weight after diet change, expressed as percentage of the original weight. ${ }^{*} P<0.05,{ }^{*} P<0.01,2$-way ANOVA. Terminal serum triglycerides (B), total cholesterol (C), and LDL (D). (E) Representative ORO-stained aortic root section in each of the 4 groups. Scale bar: $600 \mu \mathrm{m}$. The green outline represents the tunica media quantified in F. (C) Proportion of total lesion filled by a necrotic core. Each data point represents the mean value for 6 sections per mouse. The results were normalized to the $\mathrm{B} 6$ mean value in each of the 2 cohorts to account for the differences in severity between the cohorts (see Supplemental Figure 1E). (H) Number of CD45 cells in heart and aorta. (I) Frequency of monocytes and MHC- $1 \mathrm{I}^{10}$ macrophages in the $\mathrm{CD}_{4} 5^{+}$cell infiltrate in the heart. (J) Number of $\mathrm{CD} 4^{+} \mathrm{T}$ cells in the heart and aorta. (K) Frequency of CD25+ cells in CD4+ cells in the heart and aorta. (L) MFI of CD25 in the aorta on CD4+CD25+ cells. Means \pm SEM compared with $t$ tests in B, C, D, and $\mathbf{G}$ and 1-way ANOVA with Tukey's multiple-comparisons tests in $\mathbf{F}$, I, and $\mathbf{J} .{ }^{*} P<0.05,{ }^{*} P<0.01,{ }^{* *} P<0.001$. Each symbol represents 1 mouse.

Pbx1d expression in $C D 4^{+} T$ cells enhances autoimmunity in mice with dyslipidemia. Pbx1d overexpression in $\mathrm{CD}^{+} \mathrm{T}$ cells promotes autoimmune $\mathrm{T}$ cell phenotypes (25), and dyslipidemia enhances autoimmune responses in a mouse model of lupus (6). Therefore, we evaluated whether the autoimmune phenotypes associated with $\mathrm{Pbx} 1 \mathrm{~d}$ expression were enhanced by dyslipidemia. WD promoted lymphoid expansion, but the spleen weight and splenocyte numbers were higher in Pbx1d than B6 chimeric mice in the WD 
group (Figure 3, A and B). A low frequency of old CD4-Pbx1d-Tg (Pbx1d-Tg) mice produced anti-nuclear antibodies (ANA) and anti-chromatin $\operatorname{IgG}(24,25)$. Here, we show that Pbx1d expression in the $\mathrm{T}$ cells of dyslipidemic chimeric mice promoted not only earlier ANA production (Figure 3C) but also significant amounts of anti-dsDNA IgG as compared with B6 chimeric mice (Figure 3D). In contrast, the production of anti-RNA IgG, which is not part of the spontaneous autoimmune repertoire of the NZM2410 model from which Pbx1d is derived (32), was enhanced by dyslipidemia to the same extent in both Pbx1d and B6 chimeras (Supplemental Figure 1F). Interestingly, dyslipidemic Pbx1d chimeric mice also showed an increased production of IgG autoantibodies reactive to heart lysate as well as $\mathrm{ApoH}$ (also known as $\beta_{2}$-glycoprotein) and cardiolipin (Figure 3, E-G), which are associated with thrombophilia in anti-phospholipid syndrome (33). Anti-heart and -cardiolipin IgG serum levels were also higher in Pbx1d than B6 chimeric mice in the ND group (Figure 3, E and G), indicating that low levels of dyslipidemia may be sufficient for Pbx1d-overexpressing $\mathrm{T}$ cells to promote the production of anti-cardiovascular autoantibodies. Serum anti-oxLDL IgG was, however, similar between chimeric mice in the WD group (Supplemental Figure 1G). Total IgM and IgG were comparable among the groups (Supplemental Figure 1, H and I), suggesting that the global humoral response was not affected by Pbxld expressed in the dyslipidemic mice. Finally, IgG2a immune complex deposits were solely found in the glomeruli of Pbx1d chimeric mice fed with WD (Figure 3H). Glomerular immune complex deposition is a first step in lupus nephritis (34) that has not been observed in Pbx1d-Tg mice (our unpublished data). However, this phenotype, which was most likely the consequence of the increased autoantibody production, was not sufficient to progress to clinical renal pathology in the Pbx1d chimera in the WD group (data not shown). Taken together, our results suggest that the interaction between a lupus susceptibility allele, here Pbx1d expression, and dyslipidemia accelerates autoimmune responses in this model.

Dyslipidemic Pbxld chimeric mice show systemic dysregulated Teff cell and myeloid responses. Based on the known phenotypes of Pbx1d-Tg mice in control conditions (25), we focused on the relative frequency of effector memory (Tem) and naive ( $\mathrm{Tn}$ ) $\mathrm{CD}^{+} \mathrm{T}$ cell populations (Figure 4A), as well as $\mathrm{Tfh}$ and follicular regulatory $\mathrm{T}$ (Tfr) cells (Figure 4B) in the spleen. B6 chimeric mice fed with WD showed a decreased percentage of CD4 $\mathrm{T}$ cells compared with ND, which was not observed in Pbx1d chimeric mice (Figure 4C). T cell activation, measured with the early activation marker CD69, or with the increased frequency of Tem and decreased frequency of Tn populations, was greatly enhanced by WD treatment but showed no difference between strains (Figure 4, D-F). On the other hand, WD increased the frequency of Tfh cells in Pbxld but not in B6 chimeric mice (Figure 4G). Conversely, WD significantly increased the frequency of Tfr cells only in B6 chimeric mice (Figure $4 \mathrm{H}$ ). This resulted in a decreased Tfr/Tfh ratio in Pbxld chimeras fed with either diet but with no difference between diets within a strain (Figure $4 \mathrm{I}$ ). These results suggest that $\mathrm{Pbx} 1 \mathrm{~d}$ and not dyslipidemia is the major driver of the aberrant Tfh response in this model. Finally, Pbxld chimeric mice fed with WD exhibited a higher frequency of IFN- $\gamma$-producing $\mathrm{CD} 4^{+} \mathrm{T}$ cells than their counterparts fed with ND or B6 chimeric mice fed with WD (Figure 4J), indicating a synergy between Pbx1d overexpression and dyslipidemia to produce this inflammatory cytokine that aggravates atherosclerosis (35). No consistent changes in the number of $\mathrm{CD}^{+} \mathrm{T}$ cells in each subset were observed between strains because of the opposing effects of increased splenocyte numbers and decreased frequency of $\mathrm{CD} 4^{+} \mathrm{T}$ cells in the WD mice (Supplemental Figure 2, A-F).

Contrary to $\mathrm{CD}^{+} \mathrm{T}$ cells, neither Pbx1d expression nor dyslipidemia affected splenic B cells (Supplemental Figure 3, A and B). Although Pbx1d chimeric mice fed with WD presented the highest frequency of Tfh cells, the frequency and number of germinal center (GC) B cells and plasma cells were similar among the groups (Supplemental Figure 3, C and D), which parallels their similar amounts of total IgM and IgG (Supplemental Figure 1, H and I). We next investigated splenic myeloid cells in the chimeric mice (Supplemental Figure 4A), including macrophages and dendritic cells (DCs), which have recognized roles in atherosclerosis and have been identified within atherosclerotic lesions $(36,37)$. WD greatly increased the side scatter of spleen macrophages and monocytes in both Pbx1d and B6 chimeric mice (Supplemental Figure 4, B and C). However, the frequency of macrophages was the highest in Pbx1d chimeric mice fed with WD (Supplemental Figure 4B), while the frequency of monocytes was similar between WD and ND groups (Supplemental Figure 4C). There was a trend for WD to increase the frequency of conventional DCs (cDCs), but it was significant only in Pbx1d chimeric mice (Supplemental Figure 4D). Finally, the frequency of plasmacytoid DCs, a critical subset in lupus pathogenesis (38), was similar between WD and ND groups as well as between strains (Supplemental Figure 4E). Overall, the analysis of the Ldlr-KO chimeric mice revealed a synergy between Pbx1d overexpression in $\mathrm{T}$ cells and a high-fat diet for the expansion of 
A

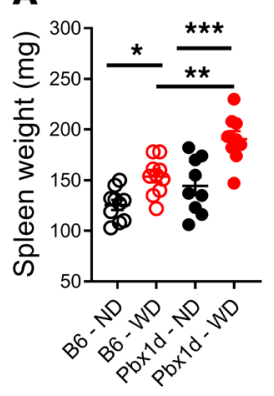

D

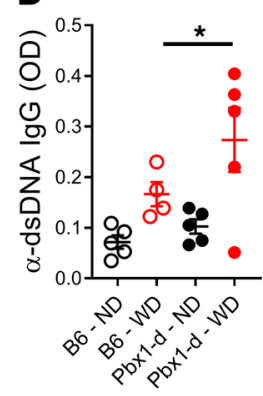

H
B

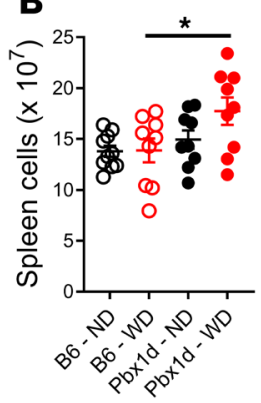

E

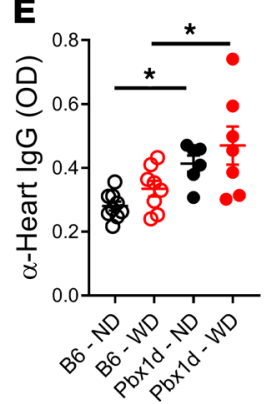

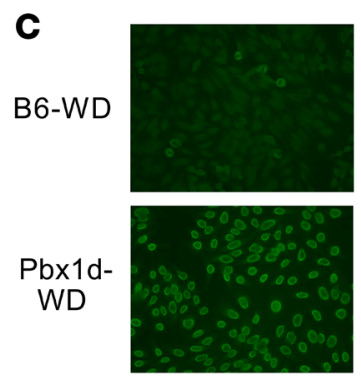

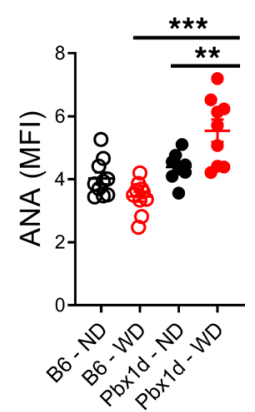

F

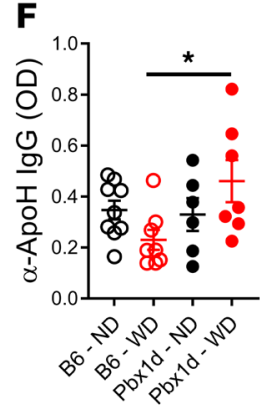

G

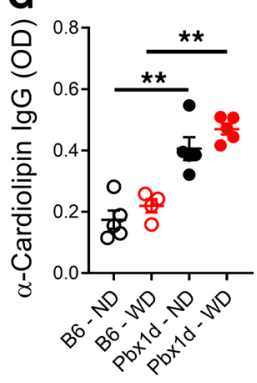

B6-ND

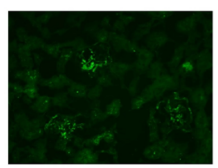

B6-WD

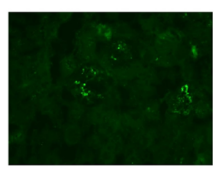

Pbx1d-ND

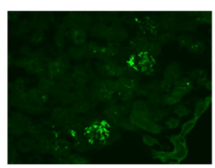

Pbx1d-WD

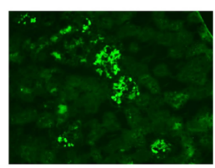

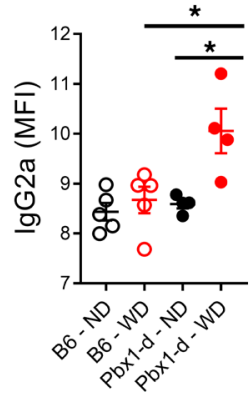

Figure 3. Pbx1d overexpression in CD4+ $T$ cells enhances autoimmunity in Ldlr-KO mice fed with WD. (A) Spleen weight and (B) total spleen cell numbers. (C-C) Serum autoantibodies at week 6 on ND or WD. Representative ANA staining and quantification of MFI (C) and anti-dsDNA (D), anti-heart (E), anti-ApoH (F), and anti-cardiolipin (G) IgG. (H) Representative IgC2a staining and MFI quantification in the kidneys of chimeric mice from each of the 4 groups. Means \pm SEM compared with 1-way ANOVA with Tukey's multiple-comparisons tests. ${ }^{*} P<0.05$, ${ }^{* *} P<0.01$, ${ }^{* * *} P<$ 0.001 . Each symbol represents 1 mouse. Original magnification, $\times 20$ (C and $\mathbf{H})$.

the frequency of Tfh cells and IFN- $\gamma^{+} \mathrm{CD} 4^{+} \mathrm{T}$ cells as well as macrophages and $\mathrm{cDCs}$, which are all cell populations that have been implicated in the pathogenesis of atherosclerosis and SLE.

Dyslipidemia alters Treg cell phenotypes in Pbxld chimeric mice. Treg dysfunction or the reduced frequency of Treg cells contributes to the phenotype of mice carrying the Slelal lupus susceptibility locus, where $P b x 1$ is located (20,21), and Pbxld overexpression in T cells directly alters the frequency of Treg cells and their induction (25). To understand the role of Pbxld in regulating Treg cells during the development of atherosclerosis, we first characterized Treg cells ex vivo in the Ldlr-KO chimeric mice. The frequency of Treg cells was increased by WD as compared with ND in $\mathrm{B} 6$ chimeric mice (Figure 5, A and B), which is consistent with a previous study reporting an increased number of Treg cells in the liver of B6 mice on WD (39). However, Pbx1d chimeric mice exhibited a similar frequency of Treg cells in the ND and WD groups, which was lower than the frequency of Treg cells in $\mathrm{B} 6$ chimeric mice in the WD group (Figure 5, A and B). The frequency of CD25 expression on Treg cells was decreased in the WD group in both strains, but the Pbx1d chimeric mice showed the lowest frequency of $\mathrm{CD} 25^{+}$ Treg cells in both WD and ND groups (Figure 5, A and C). The expression of CD62L, another marker associated with Treg suppressive function (40), was decreased in both WD and ND groups of dyslipidemic chimeric mice (Figure 5D), while cytotoxic T lymphocyte-associated protein 4 (CTLA-4) expression was decreased on the Treg cells of Pbx1d chimeric mice in the WD as compared with the ND group (Figure 5E). Finally, CD28 triggers AKT phosphorylation to activate the mTOR pathway and destabilize Foxp 3 expression (41). Expression of p-AKT was increased in Treg cells from Pbx1d chimeric mice to a greater extent than in B6 chimeric mice in 

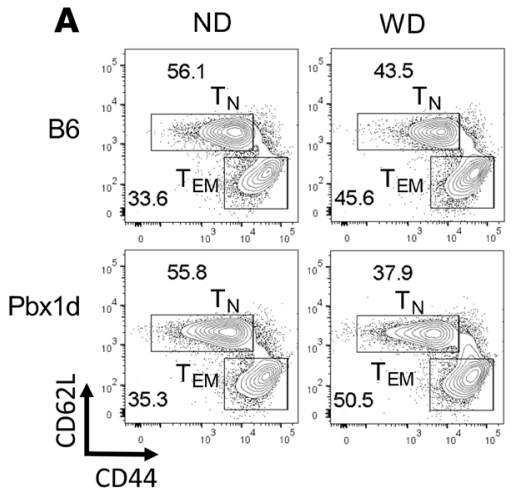

$\mathbf{B}$
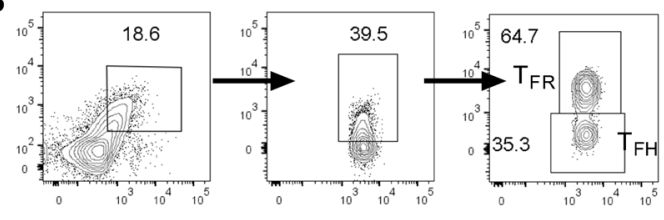

B6 - WD
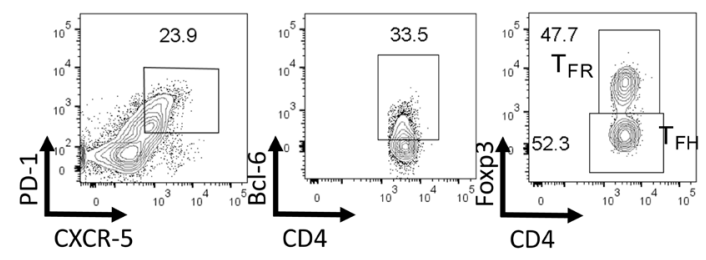

Pbx1dWD
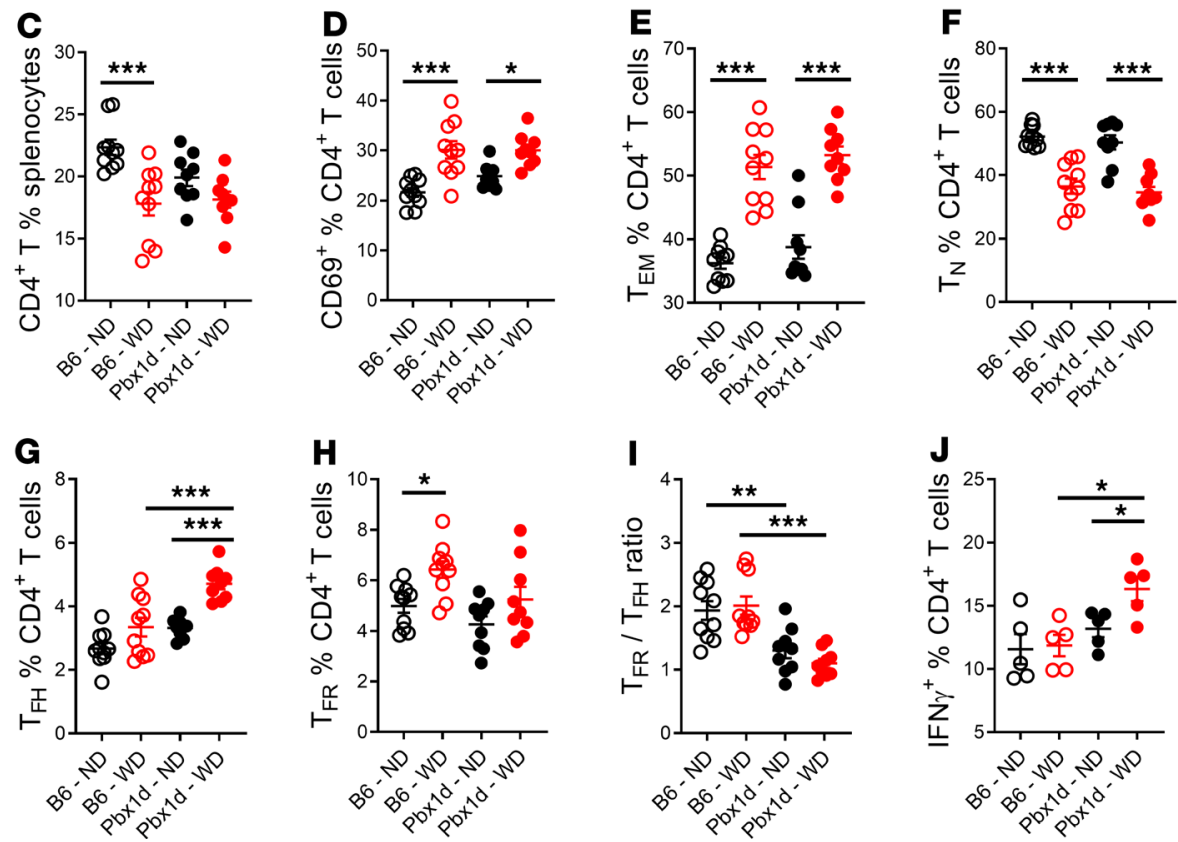

Figure 4. Pbx1d overexpression in $\mathrm{CD4}^{+} \mathrm{T}$ cells expands the Tfh and IFN- $\gamma^{+} \mathrm{CD4}^{+}$populations in LdIr-KO mice fed with WD. Representative $C D 4^{+}$-gated FACS plots showing the CD44-CD62 $\mathrm{L}^{+} \mathrm{Tn}$ and $\mathrm{CD} 44^{+} \mathrm{CD} 62 \mathrm{~L}^{-}$Tem subsets (A), as well as the CXCR5 ${ }^{+}$PD-1+Bcl6 ${ }^{+}$Foxp3 $^{-}$Tfh and CXCR5 ${ }^{+}$PD-1 ${ }^{+} \mathrm{Bcl6}^{+} \mathrm{Foxp3}^{+}$Tfr subsets (B) in the spleen of B6 and Pbx1d chimeric mice fed with ND or WD. Frequency of total splenic CD4+ $T$ cells (C); activated CD69+CD4+ $T$ cells (D); and Tem (E), $\operatorname{Tn}(\mathbf{F})$, $\operatorname{Tfh}(\mathbf{C})$, and $\operatorname{Tfr}(\mathbf{H})$ cells and $\mathrm{Tfr} / \mathrm{Tfh}$ ratio (I). (J) Frequency of IFN- $\gamma^{+} \mathrm{CD} 4^{+} \mathrm{T}$ cells. Means $\pm \mathrm{SEM}$ compared with 1-way ANOVA with Tukey's multiple-comparisons tests. ${ }^{*} P<0.05,{ }^{* *} P<0.01,{ }^{* *} P<0.001$. Each symbol represents 1 mouse.

the WD group (Figure 5F). Overall, our results suggest that Pbxld expression in T cells synergizes with dyslipidemia to impair Treg functions, which may promote atherosclerosis in the Pbxld chimeric mice fed with WD.

Pbxld overexpression impairs Treg suppressive function and differentiation under dyslipidemic conditions. To directly test whether dyslipidemia impairs the function of Pbx1d-overexpressing Treg cells, we assessed whether cholesterol affects their ability to suppress $\mathrm{CD} 4^{+} \mathrm{T}$ cell proliferation in vitro (Figure 6A). Cholesterol-methyl- $\beta$-cyclodextrin (CD-CHO) and sodium cholesteryl sulfate (CS) were used to simulate the dyslipidemia environment. $\mathrm{CD}-\mathrm{CHO}$ complexes are the most efficient manner to load cholesterol into cells in vitro (42), but it has not been used in T cells. CS activates AMPK and inhibits TCR signaling in T cells (43), which may interfere with data interpretation. Without added cholesterol, Teff cells showed a higher proliferation index in the presence of Pbx1d Treg cells, supporting that Pbx1d overexpression impaired Treg suppressive function (Figure 6, B-D). CD-CHO in vitro treatment dramatically reduced the suppressive function of Treg cells in both strains but to a greater extent in the Treg cells from Pbx1d-Tg mice (Figure 6C). Similar results were obtained with CS, although it was less dramatic than with $\mathrm{CD}-\mathrm{CHO}$ (Figure 6D). In addition, the pretreatment with CD-CHO or, to a lesser extent, with CS reduced CD25 expression on Treg cells (Figure 6, E and F), in agreement with their lower suppressive capacity. Finally, we tested whether excess cholesterol impairs $\mathrm{CD} 4^{+} \mathrm{T}$ cell 
A B6-ND B6-WD Pbx1d-ND Pbx1d-WD
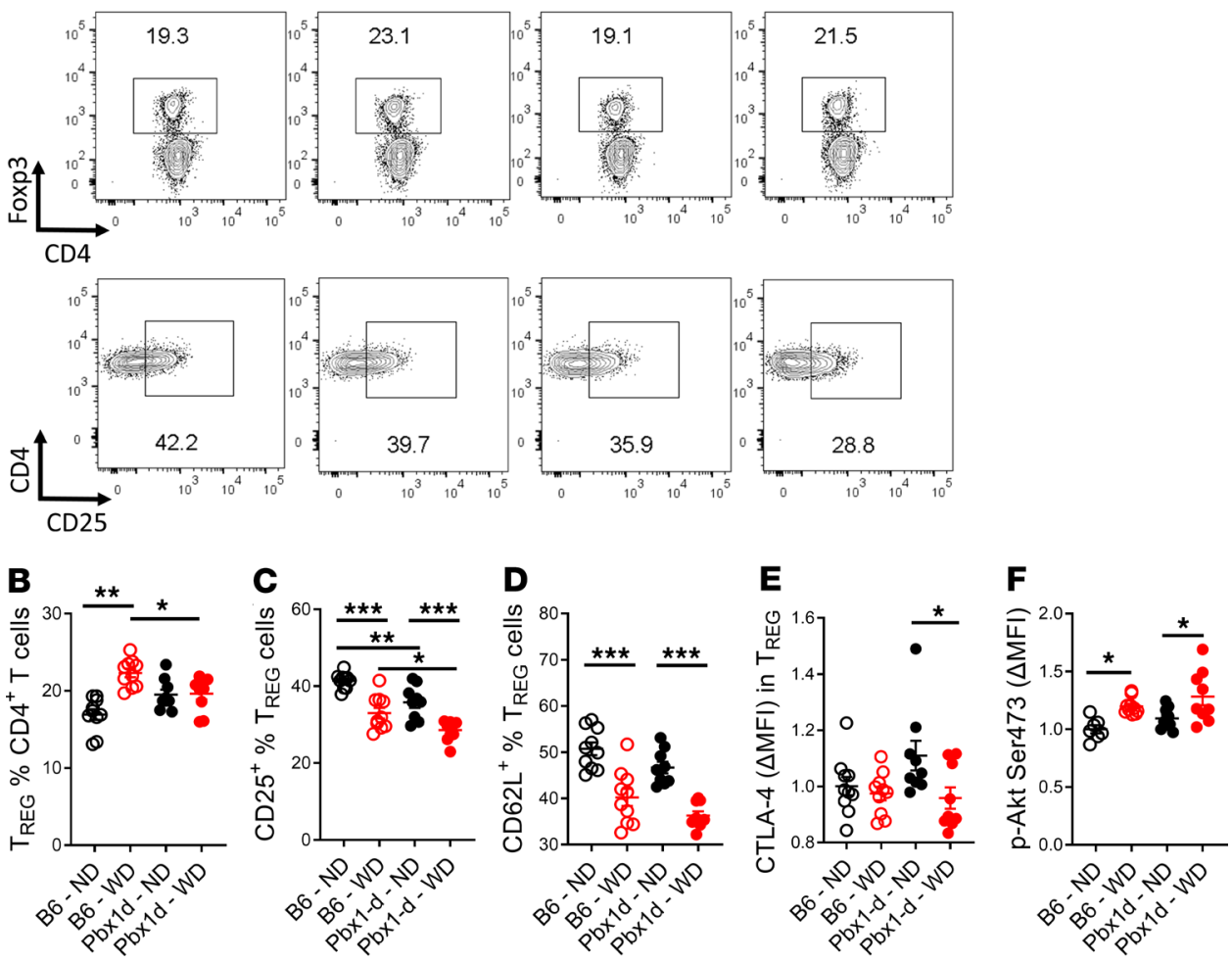

Figure 5. Pbx1d overexpression in CD4+ T cells alters Treg cell phenotypes in LdIr-KO mice fed with WD. (A) Representative $\mathrm{CD} 4^{+}$-gated FACS plots showing $\mathrm{CD}^{+}{ }^{+} \mathrm{Foxp}^{+}{ }^{+}$Treg cells and $\mathrm{CD} 4^{+}$Foxp3 $3^{+}$-gated FACS plots showing $C D 25^{\text {hi }}$ expression. Frequency of CD4+ Treg cells (B), CD25+ in Treg cells (C), and CD62 $\mathrm{L}^{+}$in Treg cells (D). (E and F) $\triangle \mathrm{MFI}$ of cytotoxic T lymphocyte-associated protein 4 (CTLA-4) and phosphorylated AKT ( $p$-AKT) Ser473 expression in CD4+Foxp3 ${ }^{+}$Treg cells. Means \pm SEM compared with 1-way ANOVA with Tukey's multiple-comparisons tests. ${ }^{*} P<0.05,{ }^{* *} P<0.01,{ }^{* * *} P$ $<0.001$. Each symbol represents 1 mouse.

polarization. CD-CHO and CS did not alter Th1 or Th17 differentiation (data not shown). CD-CHO inhibited Treg cell differentiation in both B6 and Pbx1d-Tg mice (Figure 6G), while CS had no effect (Figure 6H). Overall, these results suggest that high levels of cholesterol impair Treg cell differentiation and their function, the latter with a potentially greater impact in Pbx1d-expressing Treg cells

Cholesterol blocks Tfh cell differentiation and IL-21 production in vitro. Because Pbx1d chimeric mice fed with WD exhibited an increased frequency of Tfh cells, we next investigated directly the effect of dyslipidemia on the expansion of Tfh cells in vitro. We used B6-IL-21 $1^{\mathrm{VFP}}$ and CD4-Pbx1d-Tg-IL-21 ${ }^{\mathrm{VFP}}$ reporter mice because IL-21 is uniquely expressed by Tfh cells (44). We evaluated the expansion of Tfh cells from total CD4 ${ }^{+} \mathrm{T}$ cells under in vitro Tfh differentiation conditions, with a Th0 condition in which cells were stimulated in the absence of the cytokine cocktail as a control. A greater expansion (Figure 7, A-C) and IL-21 production (Figure 7, D-H) were obtained from Pbx1d-expressing $\mathrm{T}$ cells, which validated the assay. However, Tfh cell expansion measured with either Tfh markers or IL-21 production was inhibited by CD-CHO or CS in both strains (Figure 7, A-H). Finally, we assessed the effect of cholesterol on the maintenance of sorted CD $4^{+} \mathrm{IL}-21^{+} \mathrm{CD} 44^{+} \mathrm{T}$ cells stimulated with anti-CD3 and anti-CD28 Abs for 24 hours. In these conditions, the maintenance of IL-21 expression was impaired by CS but not by CD-CHO (Figure 7, I and J). Taken together, our results suggest that high levels of native cholesterol block Tfh cell differentiation and the maintenance of IL-21 production in vitro.

\section{Discussion}

Cardiovascular disease (CVD) is a leading comorbidity in patients with SLE, with which it shares common pathways of immune activation. Although the mechanisms responsible for the development of SLE are still largely elusive, it is important to better understand the regulatory mechanisms that bridge the 2 disease manifestations. Previous studies from our group $(7,8,13)$, as well as by others (45), have shown that the genetic susceptibility that leads to the spontaneous development of lupus in mice also aggravates the development of 
A

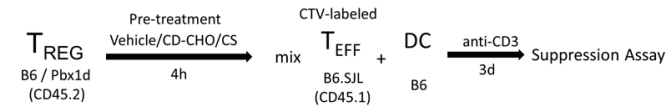

B B6 Pbx1d
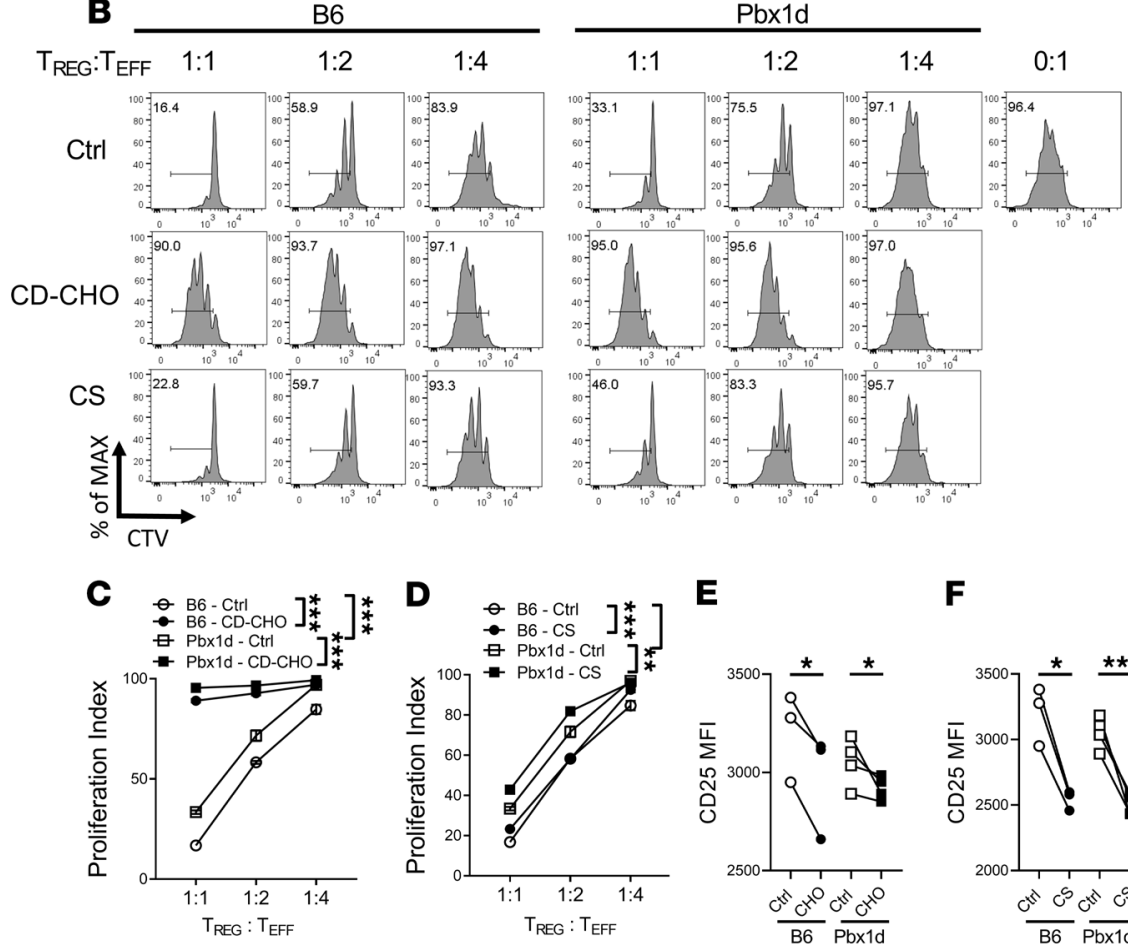

$\mathbf{E}$

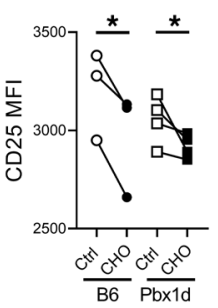

$\mathbf{F}$
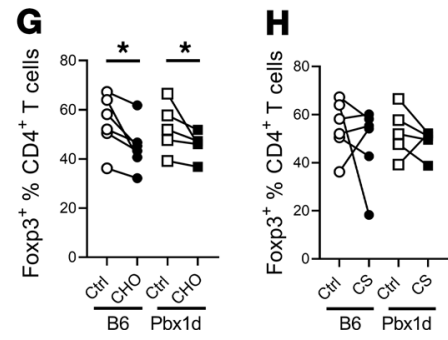

Figure 6. Cholesterol impairs Treg function in vitro. (A) Suppression assay experimental design. (B) Representative CD4 ${ }^{+} \mathrm{CD} 45.1^{+}$-gated FACS histograms showing the percentage of proliferating Teff cells with decreasing Treg/Teff ratios. Teff proliferation index in the presence of B6 or Pbx1d-Tg Treg cells pretreated with CD-CHO (C) or CS (D). Mean \pm SEM of $N=3-4$ per group compared with 2-way ANOVA; ${ }^{*} P<0.01,{ }^{* *} P<0.001$. (E and $\mathbf{F}$ ) CD25 MFI in Treg cells from B6 and Pbx1d-Tg mice after treatment with vehicle, CD-CHO, or CS for 4 hours. ( $\mathbf{G}$ and $\mathbf{H}$ ) Frequency of Foxp3 ${ }^{+}$Treg cells polarized from naive $C D 4^{+} T$ cells in the presence of $C D-C H O$ or $C S$ as compared with vehicle control after 4 days. Paired $t$ tests; ${ }^{*} P<0.05,{ }^{* *} P<0.01$. Each symbol represents 1 mouse.

atherosclerosis in dyslipidemic conditions. Using the TC mouse model of lupus, it was determined that CD4 $4^{+}$ $\mathrm{T}$ cells are responsible for the atherosclerotic effect (8). The TC mouse has been extensively used, including to map the genetic loci responsible for lupus endophenotypes, such as the production of autoreactive inflammatory $\mathrm{CD} 4^{+} \mathrm{T}$ cells (46). The first goal of this study was to map, using single congenic strains that we have developed, the genetic loci responsible for the atherosclerotic effect of the immune cells from TC mice. We focused on the Sle1 locus, which is responsible for the intrinsic autoimmune activation of $\mathrm{CD} 4^{+} \mathrm{T}$ cells $(18,20)$. Using a BM transfer model into $L d l r-\mathrm{KO}$ mice fed with a high-fat diet, we showed that immune cells expressing Sle enhanced atherosclerotic lesions similar to $\mathrm{TC}$, and increased numbers of $\mathrm{CD} 4^{+} \mathrm{T}$ cells were found in lesions of both groups. Among the Sle1 subloci, we selected the Pbx1d dominant negative allele, whose expression in T cells impairs Treg cell homeostasis and promotes Tfh cell differentiation (25), $2 \mathrm{~T}$ cell phenotypes that contribute to the pathogenesis of both lupus and atherosclerosis. Using the same BM chimera model, we showed that $\mathrm{Pbx} 1 \mathrm{~d}$ overexpression in T cells aggravated the expression of inflammatory phenotypes, of increased leukocyte infiltrates in cardiovascular tissues, of thickening of the arterial wall, and of larger necrotic cores within the 
A

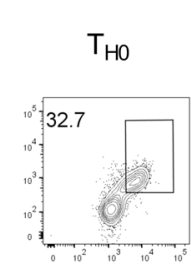

$\mathrm{Pbx} 1 \mathrm{~d}$
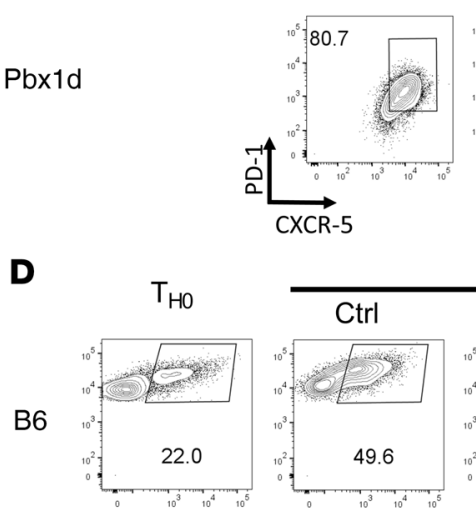

$\mathrm{T}_{\mathrm{FH}}$

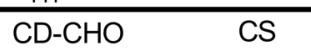

Pbx1d
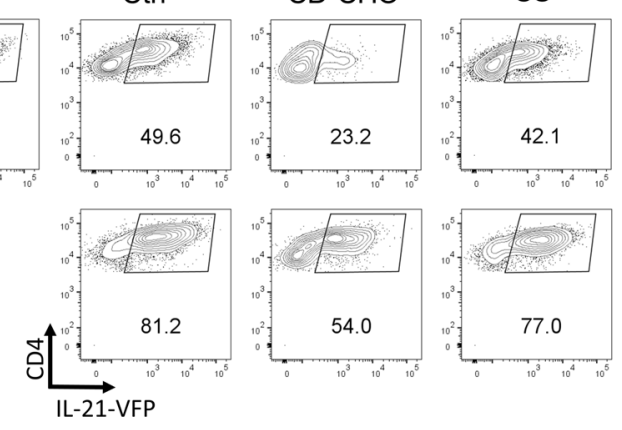

$\mathbf{G}$

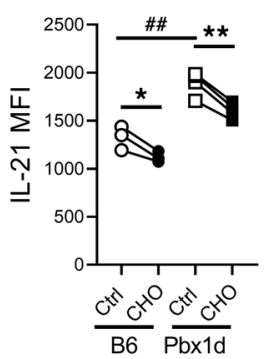

H

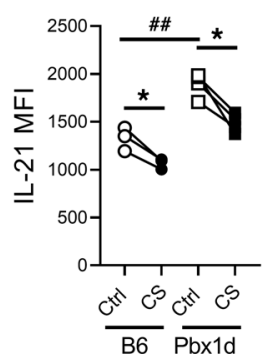

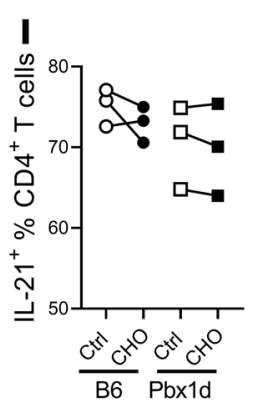

B
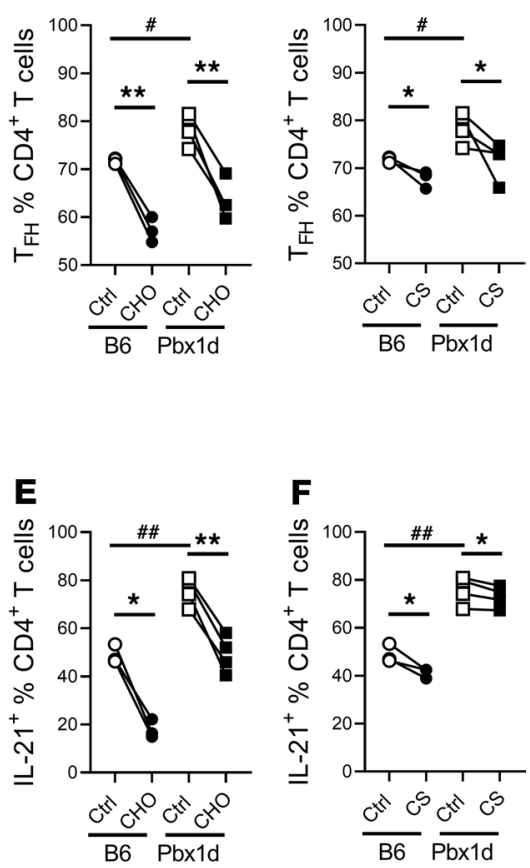

Figure 7. Cholesterol reduces Tfh cell expansion in vitro. (A) Representative CD4+-gated FACS plots showing the

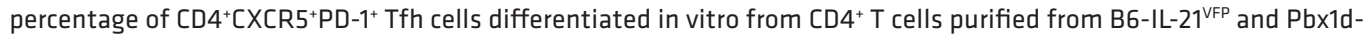
Tg-IL-21 ${ }^{\mathrm{VFP}}$ mice in the presence of vehicle, CD-CHO, or CS. (B and C) Frequency of Tfh cells corresponding to the assay shown in A. (D) Representative CD4+-gated FACS plots showing the percentage of IL-21+CD4+ $\mathrm{T}$ cells differentiated in vitro from B6-IL-21 ${ }^{\mathrm{VFP}}$ and Pbx1d-Tg-IL-21 VFP $C D 4^{+} \mathrm{T}$ cells in the presence of vehicle, CD-CHO, or CS. Frequency of

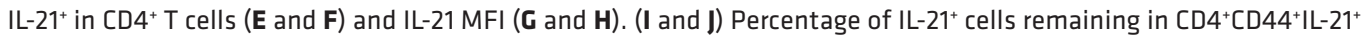
cells isolated from B6-IL-21 VFP and Pbx1d-Tg-IL-21 VFP mice after 24 hours' stimulation with anti-CD3 and anti-CD28 antibodies in the presence of vehicle, CD-CHO, or CS. Paired $t$ tests; ${ }^{*} P<0.05$, ${ }^{*} P<0.01$, unpaired $t$ test; ${ }^{*} P<0.05$, $\# \#<0.01$. Each symbol represents 1 mouse.

lesions, which have been associated with the severity of atherosclerotic lesions. Moreover, Pbx1d overexpression in $\mathrm{CD} 4^{+} \mathrm{T}$ cells promoted autoimmune $\mathrm{T}$ cell phenotypes in dyslipidemic mice. This was associated with expansion of numbers of Th cells as well as altered expression of Treg markers associated with suppressive function. High levels of cholesterol impaired the differentiation and suppressive function of Treg cells from either Pbx1d-Tg mice or B6 controls in vitro. In vitro assays, however, did not support that high cholesterol promotes the expansion or the maintenance of Tfh cells. This might be explained by the fact that Treg cells have a wide distribution within lymphoid tissue (47), where they may directly encounter cholesterol, while pre-Tfh and Tfh cells are mostly in the B cell zone or deep within GCs, respectively. Moreover, the increased frequency of Tfh cells in dyslipidemic Pbxld chimeric mice may reflect that exposure to oxidized lipids rather than native cholesterol skews T cell effector functions. The specific contribution of Treg and Tfh cells will need to be addressed directly in $L d l r$-KO chimeras with Pbx1d expression specific to each of these 2 subsets. 
Overall, this study suggests that a single lupus susceptibility gene enhances both autoimmune and atherosclerotic pathogenesis through impaired Treg cell functions.

Immune cells participate decisively in all stages of atherosclerosis. Among them, $\mathrm{T}$ lymphocytes are influenced by inflammatory signals and contribute to the onset and progression of atherosclerosis. Tfh cells have been recently scrutinized in autoimmune diseases and CVD. Tfh cells are found in large numbers in lupus-prone mice (48) and in the peripheral blood of patients with lupus, with a correlation with disease activity (49). Tfh cells promote atherosclerosis in mice, with dyslipidemia inducing Tfh differentiation from unstable "ex-Treg" cells (50) or through IL-27 produced by DCs (6). A direct contribution of Tfh cells to atherosclerosis is shown by the reduced disease severity induced by Bcl6-deficient CD4+ $\mathrm{T}$ cells (50). On the other hand, transferring BM from lupus-prone mice into dyslipidemic mice not only increased the severity of atherosclerotic lesions but also enhanced their Tfh and GC responses (6), again suggesting synergy between SLE and CVD through the adaptive immune system. Here, we also found an increased frequency of Tfh cells in the Pbx1d chimeric mice fed with a high-fat diet as compared with either B6 chimeric mice on the same high-fat diet or Pbx1d chimeric mice on a control diet. This expansion of the Tfh subset was associated with increased autoantibody production and renal immune complex deposition. At steady state, however, Pbx1d is not sufficient to expand the number of Tfh cells and has only a modest autoimmune effect in aged mice (25). These results indicate that a gene/environment interaction between Pbx1d and dyslipidemia enhances the Tfh response in this BM chimeric Ldlr-KO model. We were not able to show in vitro that cholesterol favors Tfh cell differentiation or maintenance. This may reflect the complex multicellular environment that is involved in the process and that may not have been reproduced in the assay. Cholesterol may not act directly on Tfh cells but on DCs or GC B cells. Alternatively, cholesterol may not be the metabolite that alters Tfh cell fate in dyslipidemic mice.

In the context of cholesterol homeostasis, Treg cells have opposite functions to other T cell subsets (51). Treg cells are functionally impaired in both mice and humans with coronary artery disease, although their numbers initially increase in early lesions, then decrease over time with disease progression $(10,52)$. Treg cells also inhibit endothelial cell activation, lymphocyte infiltration, foam cell formation, and inflammatory macrophage differentiation $(51,53)$. Treg cell deficiency increases the atherosclerotic lesions in $L d l r-\mathrm{KO}$ mice (11). Moreover, transferring BM from Treg-depleted mice increases plasma cholesterol levels in Ldlr-KO mice (9), indicating an atheroprotective role of Treg cells. In addition, intracellular cholesterol homeostasis affects Treg cell development, and limiting cholesterol efflux by deleting ATP-binding cassette transporter G1 increased Treg cell differentiation (54). In this study, we observed an increased frequency of splenic Treg cells in chimeric mice on WD, likely in response to inflammation. The Pbx1d chimeric mice fed with high fat showed, however, a lower frequency of Treg cells, and their expression of most Treg cell functional markers was impaired. Similar results were observed in the aorta, which corresponded to a higher number of $\mathrm{CD}^{+} \mathrm{T}$ cell infiltrates in the surrounding myocardium. This indicated that Pbx1d affects both Treg cell frequency and function in this model. In vitro, cholesterol impaired both Treg differentiation and suppressive function. Taken together, our findings indicate again a gene/environment interaction between $\mathrm{Pbx} 1 \mathrm{~d}$ and dyslipidemia that impaired Treg cells and consequently promoted both atherosclerosis and autoimmunity.

Innate cells also participate in the development of atherosclerosis. Dysregulation of macrophage and monocyte phenotypes is a major driver of atherosclerosis, in which macrophages and monocytes are exposed to inflammatory cytokines, oxidized lipids, and cholesterol crystals (55). We observed increased frequency and number of splenic macrophages in the Pbxld chimeric mice fed with a high-fat diet. In the cardiovascular tissues, similar numbers of innate immune cells were observed in Pbx1d and B6 chimeric mice fed with a high-fat diet. However, we observed that the combination of Pbx1d expression in $\mathrm{T}$ cells and dyslipidemia expanded the population of $\mathrm{MHC}-\mathrm{II}^{\mathrm{Io}}$ macrophages, which have been described as cardiac-resident macrophages that are uniquely proficient at processing dead cardiomyocytes (30). Future studies are required to determine whether this phenotype is a mere consequence of increased tissue injury, including necrotic cores, or whether $\mathrm{Pbx} 1 \mathrm{~d}$-overexpressing $\mathrm{T}$ cells in a dyslipidemic milieu directly promote the expansion of these cells, which in turn contribute to tissue injury. We did not find any difference in B cell phenotypes, either in the spleen or in cardiovascular tissue. Overall, these results show that autoimmune $\mathrm{CD}^{+} \mathrm{T}$ cells are a major contributor to lupus atherosclerosis, at least in the model we used.

In conclusion, we provide evidence for the first time to our knowledge that a lupus susceptibility allele, Pbxld, overexpressed in $\mathrm{CD}^{+} \mathrm{T}$ cells enhances phenotypes associated with atherosclerosis severity and that dyslipidemia enhances the autoimmune phenotypes this allele induces. Furthermore, we show that interaction 
between $\mathrm{Pbx} 1 \mathrm{~d}$ expression and dyslipidemia altered Treg cell differentiation and functions. These findings provide a genetic and functional link that may bridge autoimmune disease and CVD.

\section{Methods}

Mice. C57BL/6J (B6), B6.SJL-Ptprc ${ }^{\mathrm{a}} P e c^{\mathrm{b}} /$ BoyJ (B6.SJL), B6-Foxp3 ${ }^{\text {YFPCre }}$, and B6.129S7-Ld1r ${ }^{\mathrm{tm} 1 \mathrm{Her}} / \mathrm{J}$ (Ldlr$\mathrm{KO})$ mice were originally bought from the Jackson Laboratory. TC, B6.Sle1, and B6.Sle3 mice have been previously described $(12,56)$. CD4-Pbx1d-Tg (Pbx1d-Tg) mice were generated at the University of Florida (UF) transgenic core as previously described (25). Pbx1d-Tg mice were bred with B6.Foxp3 ${ }^{\text {YFPCre }}$ mice to generate the Treg cell reporter strain, Pbx1d-Tg.Foxp3 ${ }^{\text {YFPCre }}$. For in vitro Tfh experiments, Pbx1d-Tg mice were bred with B6.IL-21 ${ }^{\mathrm{VFP}}$ mice (57) to generate the IL-21 reporter strain, Pbx1d-Tg.IL-21 ${ }^{\mathrm{VFP}}$.

$B M$ chimeras. Briefly, BM cells were isolated from 2-month-old donor mice. After T cells were depleted with anti-CD5 microbeads (Miltenyi Biotec), BM cells were i.v. injected into 3-month-old Ldlr-KO recipient mice that were irradiated with 2 doses of 4.25-4.50 Gy, 4 hours apart, before they received $10^{7} \mathrm{BM}$ cells from either donor. After BM transfer, $L d l r$-KO mice were treated with antibiotics (Baytril, Bayer) for 10 days. After 6 or 16 weeks of reconstitution, chimeric $L d l r$-KO mice were fed a WD (Envigo TD.88137, $42 \%$ calories from fat) or ND (Envigo 7912) for another 8 weeks before sacrifice. Mice were fasted for 4 hours before serum was collected after the diet change every other week. Each Vanderbilt University (VU) and UF BM chimera experiment was performed on 2 independent cohorts. In the VU experiment, all mice were fed a WD. The UF experiment was performed with 4 groups: Pbx1d-Tg.Foxp3 ${ }^{\text {YFPCre } W D}$ or ND and B6.Foxp $3^{\text {YFPCre }} \mathrm{WD}$ or $\mathrm{ND}$, according to the BM donor strain of origin and the diet.

Histology. The presence of IgG2a/C3 immune complex in the glomeruli was detected in frozen kidney sections with fluorescently tagged anti-IgG2a and anti-C3 Abs, as previously reported (58), and quantitated with ImageJ (NIH). Atherosclerosis in the aortic sinus was quantified as previously described (7). Briefly, 6 consecutive $10-\mu \mathrm{m}$ sections were stained for 4 hours at room temperature with ORO. Lipid staining area and necrotic cores were quantified with Aperio (Leica Biosystems). $\mathrm{CD}^{+} \mathrm{T}$ cells were quantified in aortic sinus by staining 5 - $\mu \mathrm{m}$ sections serial sections with biotin-conjugated rat anti-CD4 mAb (clone RM4-5, BD Biosciences) and Texas red-conjugated streptavidin (Vector Laboratories). Numbers of $\mathrm{CD}^{+} \mathrm{T}$ cells and total cells in lesions were counted. Data were expressed as $\mathrm{CD} 4^{+} \mathrm{T}$ cells as a percentage of total cells in lesions. Pathology was scored semiquantitatively on a $0-3$ scale for 4 parameters on H\&E sections: aortic valve histiocytic inflammation, perivalvular chronic inflammation, apoptotic cells, and perivalvular fibrosis, which were totaled to obtain a single score per study subject. All histology assessments were performed in a blinded fashion.

ELISA. Anti-heart IgG was detected on Immulon $2 \mathrm{HB}$ plates (Thermo Fisher Scientific) precoated with $1 \mu \mathrm{g} / \mathrm{mL}$ rat heart lysate (Novus Biologicals) in PBS, washed, and blocked with $0.1 \%$ gelatin and $3 \% \mathrm{BSA}$ in $3 \mathrm{mM}$ EDTA. Serum was diluted at $1: 50$ in dilution buffer $(0.1 \%$ gelatin, $2 \%$ BSA, $3 \mathrm{mM}$ EDTA, and $0.05 \%$ Tween-20) and incubated at $37^{\circ} \mathrm{C}$ for 1 hour. Goat anti-mouse IgG-alkaline phosphatase (AP308A, MilliporeSigma) was used as a secondary Ab at a 1:1000 dilution. Alkaline phosphatase activity was detected with para-nitrophenyl phosphate substrate in $0.025 \mathrm{M}$ bicarbonate buffer with $1 \mathrm{M}$ $\mathrm{MgCl}_{2}$ as previously described (59). The same ELISA protocol was used with $1 \mu \mathrm{g} / \mathrm{mL}$ recombinant $\beta_{2}$ glycoprotein/ApoH (R\&D Systems, Bio-Techne) to detect anti-phospholipid (anti-ApoH) IgG, with $30 \mu \mathrm{g} /$ mL RNA isolated from mouse splenocytes to detect anti-RNA IgG and with $75 \mu \mathrm{g} / \mathrm{mL}$ cardiolipin (MilliporeSigma) in ethanol for anti-cardiolipin IgG ELISA as previously described (60). Anti-dsDNA IgG was detected as previously described with sera diluted 1:100 (61). For ANA detection, serum was diluted 1:40 and used for indirect immunostaining of Hep-2 slides (Bio-Rad) with Alexa Fluor 488-conjugated goat anti-mouse IgG (BD Biosciences). Slides were fixed with Fluoromount (MilliporeSigma), and fluorescence intensity was quantified with the ImageJ software. For anti-oxLDL IgG ELISA, MaxiSorp plates (Nunc) were coated with $10 \mu \mathrm{M}$ oxLDL (MilliporeSigma) at $4^{\circ} \mathrm{C}$ overnight. Briefly, plates were washed in PBS containing $0.05 \%$ Tween, then blocked in 1\% BSA/PBS (Thermo Fisher Scientific) at room temperature for 2 hours. Plates were then washed in PBS-Tween, and serum samples diluted in 1\% BSA/PBS were incubated for 2 hours at room temperature. Anti-mouse IgG-HRP or IgM-HRP (1:5000) (Promega) was added to the wells and incubated overnight at $4^{\circ} \mathrm{C}$. Plates were washed and OptEIA TMB substrate (BD Biosciences) was added. Plates were developed for 10 minutes prior to quenching with $2 \mathrm{M} \mathrm{HCl}$ and read at $450 \mathrm{nM}$ as previously described (62). Triglycerides and cholesterol were measured using a commercially available kit (Raichem), and LDL and HDL were measured by HDL and LDL/VLDL Quantitation Kit (MilliporeSigma). All the protocols followed manufacturers' instructions. 
Flow cytometry. Single-cell suspensions were prepared using standard procedures from spleens. Cells were stained in FACS staining buffer (2.5\% FBS, 0.05\% sodium azide in PBS). Fluorochrome-conjugated Abs are as follows: B220 (RA3-6B2), Bc1-6 (K112-91), CD3e (145-2C11), CD45.1 (A-20), CD62L (MEL-14), CD95 (Jo2), CXCR5 (2G8), IgD (217-170), and Ly6C (AL-21) were purchased from BD Biosciences. CD4 (RM4-5), CD138 (281-2), CTLA-4 (UC10-4B9), and IFN- $\gamma$ (XMG1.2) were purchased from BioLegend. CD4 (GK1.5), CD44 (IM7), CD25 (PC61.5), CD69 (H1.2F3), Foxp3 (FJK-16S), GL-7 (GL-7), GITR (DTA-1), p-AKT (SDRNR), PD-1 (RMP1-30), and PDCA-1 (eBio927) were purchased from eBioscience, Thermo Fisher Scientific. CD11b (M1/70) and CD11c (HL3) were purchased from STEMCELL Technologies. F4/80 (BM8) was purchased from Santa Cruz Biotechnology. MHCII (M5/114.15.2) was purchased from Miltenyi Biotec. Follicular T cells were stained in a 3-step process using purified anti-CXCR5 mAb (2G8), followed with biotinylated anti-rat IgG (112065-167, Jackson ImmunoResearch) and PerCP-Cy5.5 streptavidin (551419, BD Biosciences) in FACS on ice. All dead cells were excluded with fixable viability dye (eFluor780; Thermo Fisher Scientific). Intracellular staining was performed with a fixation/permeabilization kit (eBioscience, Thermo Fisher Scientific). For cytokine detection, splenocytes were stimulated with Leukocyte Activation Cocktail (BD Biosciences) for 4 hours. Aortas were processed for FACS analysis as previously described with modifications (63). Briefly, after heart perfusion, the whole aorta and bottom half of the heart (the upper half being used for histology) were cut into small pieces with fine scissors in $1 \mathrm{~mL}$ digestion buffer containing collagenase $\mathrm{A}(1 \mathrm{mg} / \mathrm{mL})$, collagenase $\mathrm{B}(1 \mathrm{mg} / \mathrm{mL})$ and DNase I $100 \mu \mathrm{g} / \mathrm{mL}$ (all from MilliporeSigma) in RPMI 1640 supplemented with 5\% FBS. Samples were incubated at $37^{\circ} \mathrm{C}$ under continuous rotation for 30 minutes. The digestion was stopped by adding 5 times the volume of cold RPMI with 5\% FBS, and then samples were filtered through a $40-\mu \mathrm{m}$ strainer. Cells were washed and resuspended in FACS staining buffer for counting and staining. All samples were acquired on an LSRFortessa flow cytometer (BD Biosciences) and analyzed with FlowJo software (Tree Star) as previously described (64).

Treg cell suppression assay. CD $45.1^{+} \mathrm{CD} 4{ }^{+} \mathrm{CD} 25^{-}$Teff cells were isolated with the $\mathrm{CD} 4{ }^{+} \mathrm{CD} 25^{+} \mathrm{T}$ cell isolation kit (Miltenyi Biotec) from B6.SJL congenic mice and labeled with Cell Trace Violet (CTV) (Life Technologies, Thermo Fisher Scientific). DCs were isolated from B6 mice with anti-CD11c microbeads. $\mathrm{CD} 4^{+} \mathrm{CD} 25^{+}$Treg cells were isolated from B6.Foxp3 $3^{\mathrm{YFPCr}}$ and Pbx1d-Tg.Foxp3 ${ }^{\mathrm{YFPCre}}$ mice and pretreated with PBS, $100 \mu \mathrm{M}$ CD-CHO (MilliporeSigma), or $50 \mu \mathrm{M}$ CS (MilliporeSigma) in complete RPMI (cRPMI) medium for 4 hours. Treg cells were washed and incubated with Teff cells $(6.6 \times$ $\left.10^{4}\right)$ at a $1: 1$ to $1: 4$ ratio in the presence of DCs $\left(10^{4}\right.$ cells) and soluble anti-CD3e $\mathrm{Ab}(1 \mu \mathrm{g} / \mathrm{mL})$ for 3 days. The proliferation of CD $45.1^{+} \mathrm{CD} 4^{+} \mathrm{CD} 25^{-}$Teff cells was determined by dilution of CTV, and the proliferation index was calculated with FlowJo software.

Treg and Tfh cell differentiation. $\mathrm{CD} 4{ }^{+} \mathrm{CD} 25^{-} \mathrm{T}$ cells were isolated from B6.Foxp $3^{\text {YFPCre }}$ and Pbx1d-Tg. Foxp3 $3^{\text {YFPCre }}$ mice. The Th0 condition corresponds to a stimulation with precoated $2 \mu \mathrm{g} / \mathrm{mL}$ anti-CD3 (1452C11, BD Biosciences) and soluble $1 \mu \mathrm{g} / \mathrm{mL}$ anti-CD28 Ab (37.51, BD Biosciences). The Treg polarizing conditions were as follows: $10^{6} \mathrm{CD} 4^{+} \mathrm{CD} 25^{-} \mathrm{T}$ cells were incubated in Th0 condition in addition to $3 \mathrm{ng} /$ mL TGF- $\beta$ (PeproTech), 50 ng/mL IL-2 (R\&D Systems, Bio-Techne) and $10 \mu \mathrm{g} / \mathrm{mL}$ anti-IFN- $\gamma$ (XMG1.2, Bio X Cell) and anti-IL-4 (11B11, Bio X Cell) Abs in cRPMI medium for 4 days (64). For Tfh cell differentiation, $1 \times 10^{6} \mathrm{CD} 4^{+} \mathrm{T}$ cells isolated from B6.IL-21 ${ }^{\mathrm{VFP}}$ and Pbx1d-Tg.IL-21 $1^{\mathrm{VFP}}$ mice incubated in Th0 condition plus $100 \mathrm{ng} / \mathrm{mL}$ IL-6 (BioLegend); 50 ng/mL IL-21 (PeproTech); 10 g/mL anti-IL-2 (JES6-1A12, BioLegend), anti-IFN- $\gamma$, and anti-IL-4; and $5 \mu \mathrm{g} / \mathrm{mL}$ anti-TGF- $\beta$ (1D11, R\&D Systems, Bio-Techne) Abs in cRPMI medium for 3 days (65). In some samples for all 3 conditions, $100 \mu \mathrm{M}$ CD-CHO or $50 \mu \mathrm{M}$ CS was added to the polarizing media.

Statistics. Statistical analyses were performed with GraphPad Prism 8.0 software. Differences between groups were evaluated by 1-way/2-way ANOVA with Tukey's multiple-comparisons tests, or unpaired or paired $t$ tests, as indicated in the text. All tests were 2 tailed. The results are expressed as means \pm SEM. The levels of statistical significance were set at ${ }^{*} P<0.05$, ${ }^{* *} P<0.01$, and ${ }^{* * *} P<0.001$.

Study approval. All experiments were conducted according to protocols approved by VU and UF Institutional Animal Care and Use Committees.

\section{Author contributions}

WL, AM, and LM designed the experiments. WL, ASE, and CC conducted in vivo experiments. WL, ASE, XT, and JB conducted in vitro experiments. LZS performed tissue processing and histology. GA, SCC, XZ, BR, and KF participated in experimental design and data interpretation. LM and AM supervised the study. WL, BR, AM, and LM wrote the manuscript. 


\section{Acknowledgments}

We thank all the Morel lab members for technical assistance and discussion and the UF Department of Pathology Molecular Pathology Core for their assistance with histology. This work is supported by a grant from the NIH R01 AI045050-21 to LM.

Address correspondence to: Laurence Morel, PO Box 0275, University of Florida, Gainesville, Florida 32610-0275, USA. Phone: 352.392.3790; Email: morel@ufl.edu.

1. Libby P, Ridker PM, Hansson GK. Progress and challenges in translating the biology of atherosclerosis. Nature. 2011;473(7347):317-325.

2. Roman MJ, et al. Prevalence and correlates of accelerated atherosclerosis in systemic lupus erythematosus. $N$ Engl J Med. 2003;349(25):2399-2406.

3. Katz G, Smilowitz NR, Blazer A, Clancy R, Buyon JP, Berger JS. Systemic lupus erythematosus and increased prevalence of atherosclerotic cardiovascular disease in hospitalized patients. Mayo Clin Proc. 2019;94(8):1436-1443.

4. Suárez-Fueyo A, Bradley SJ, Tsokos GC. T cells in systemic lupus erythematosus. Curr Opin Immunol. 2016;43:32-38.

5. Simons KH, de Jong A, Jukema JW, de Vries MR, Arens R, Quax PHA. T cell co-stimulation and co-inhibition in cardiovascular disease: a double-edged sword. Nat Rev Cardiol. 2019;16(6):325-343.

6. Ryu H, et al. Atherogenic dyslipidemia promotes autoimmune follicular helper T cell responses via IL-27. Nat Immunol. 2018;19(6):583-593.

7. Stanic AK, et al. Immune dysregulation accelerates atherosclerosis and modulates plaque composition in systemic lupus erythematosus. Proc Natl Acad Sci U S A. 2006;103(18):7018-7023.

8. Wilhelm AJ, Rhoads JP, Wade NS, Major AS. Dysregulated CD4+ T cells from SLE-susceptible mice are sufficient to accelerate atherosclerosis in LDLr-/- mice. Ann Rheum Dis. 2015;74(4):778-785.

9. Klingenberg R, et al. Depletion of FOXP3 + regulatory T cells promotes hypercholesterolemia and atherosclerosis. J Clin Invest. 2013;123(3):1323-1334.

10. Mor A, et al. Role of naturally occurring CD4+ CD25+ regulatory T cells in experimental atherosclerosis. Arterioscler Thromb Vasc Biol. 2007;27(4):893-900.

11. Ait-Oufella H, et al. Natural regulatory T cells control the development of atherosclerosis in mice. Nat Med. 2006;12(2):178-180.

12. Morel L, et al. Genetic reconstitution of systemic lupus erythematosus immunopathology with polycongenic murine strains. Proc Natl Acad Sci U S A. 2000;97(12):6670-6675.

13. Braun $\mathrm{Na}$, Wade NS, Wakeland EK, Major AS. Accelerated atherosclerosis is independent of feeding high-fat diet in systemic lupus erythematosus-susceptible LDLr(-/-) mice. Lupus. 2008;17(12):1070-1078.

14. Mohan C, Yu Y, Morel L, Yang P, Wakeland EK. Genetic dissection of Sle pathogenesis: Sle3 on murine chromosome 7 impacts T cell activation, differentiation, and cell death. J Immunol. 1999;162(11):6492-6502.

15. Wade NS, Stevenson BG, Dunlap DS, Major AS. The lupus susceptibility locus Sle3 is not sufficient to accelerate atherosclerosis in lupus-susceptible low density lipoprotein receptor-deficient mice. Lupus. 2010;19(1):34-42.

16. Mohan C, Morel L, Yang P, Wakeland EK. Genetic dissection of systemic lupus erythematosus pathogenesis: Sle2 on murine chromosome 4 leads to B cell hyperactivity. J Immunol. 1997;159(1):454-465.

17. Morel L, Tian XH, Croker BP, Wakeland EK. Epistatic modifiers of autoimmunity in a murine model of lupus nephritis. Immunity. 1999;11(2):131-139.

18. Sobel ES, Satoh M, Chen Y, Wakeland EK, Morel L. The major murine systemic lupus erythematosus susceptibility locus Sle1 results in abnormal functions of both B and T cells. J Immunol. 2002;169(5):2694-2700.

19. Morel L, Blenman KR, Croker BP, Wakeland EK. The major murine systemic lupus erythematosus susceptibility locus, Sle1, is a cluster of functionally related genes. Proc Natl Acad Sci U S A. 2001;98(4):1787-1792.

20. Chen Y, Cuda C, Morel L. Genetic determination of T cell help in loss of tolerance to nuclear antigens. J Immunol. 2005;174(12):7692-7702.

21. Cuda CM, Wan S, Sobel ES, Croker BP, Morel L. Murine lupus susceptibility locus Sle1a controls regulatory T cell number and function through multiple mechanisms. J Immunol. 2007;179(11):7439-7447.

22. Asahara H, Dutta S, Kao HY, Evans RM, Montminy M. Pbx-Hox heterodimers recruit coactivator-corepressor complexes in an isoform-specific manner. Mol Cell Biol. 1999;19(12):8219-8225.

23. Sengupta M, Liang S, Potula HH, Chang LJ, Morel L. The SLE-associated Pbx1-d isoform acts as a dominant-negative transcriptional regulator. Genes Immun. 2012;13(8):653-657.

24. Cuda CM, et al. Pre-B cell leukemia homeobox 1 is associated with lupus susceptibility in mice and humans. J Immunol. 2012;188(2):604-614.

25. Choi SC, et al. The lupus susceptibility gene $\mathrm{Pbx} 1$ regulates the balance between follicular helper $\mathrm{T}$ cell and regulatory $\mathrm{T}$ cell differentiation. J Immunol. 2016;197(2):458-469.

26. Sobel ES, Morel L, Baert R, Mohan C, Schiffenbauer J, Wakeland EK. Genetic dissection of systemic lupus erythematosus pathogenesis: evidence for functional expression of Sle3/5 by non-T cells. J Immunol. 2002;169(7):4025-4032

27. Xu Z, Butfiloski EJ, Sobel ES, Morel L. Mechanisms of peritoneal B-1a cells accumulation induced by murine lupus susceptibility locus Sle2. J Immunol. 2004;173(10):6050-6058.

28. Xue-Mei L, Jie C, Xuan D, Xiao-Xing L, Chun-Lin H, Yu-Jie L. Changes in CD $4^{+}$CD25+ Tregs in the pathogenesis of atherosclerosis in ApoE ${ }^{-/}$mice. Exp Biol Med (Maywood). 2017;242(9):918-925.

29. Thorp E, Tabas I. Mechanisms and consequences of efferocytosis in advanced atherosclerosis. J Leukoc Biol. 2009;86(5):1089-1095

30. Epelman S, et al. Embryonic and adult-derived resident cardiac macrophages are maintained through distinct mechanisms at steady state and during inflammation. Immunity. 2014;40(1):91-104. 
31. Horwitz DA. Identity of mysterious CD4+CD25-Foxp3+ cells in SLE. Arthritis Res Ther. 2010;12(1):101.

32. Mohan C, Alas E, Morel L, Yang P, Wakeland EK. Genetic dissection of SLE pathogenesis. Sle1 on murine chromosome 1 leads to a selective loss of tolerance to H2A/H2B/DNA subnucleosomes. J Clin Invest. 1998;101(6):1362-1372.

33. Chock YP, Moulinet T, Dufrost V, Erkan D, Wahl D, Zuily S. Antiphospholipid antibodies and the risk of thrombocytopenia in patients with systemic lupus erythematosus: a systematic review and meta-analysis. Autoimmun Rev. 2019;18(11):102395.

34. Davidson A, Aranow C. Pathogenesis and treatment of systemic lupus erythematosus nephritis. Curr Opin Rheumatol. 2006;18(5):468-475.

35. Ivashkiv LB. IFN $\gamma$ : signalling, epigenetics and roles in immunity, metabolism, disease and cancer immunotherapy. Nat Rev Immunol. 2018;18(9):545-558.

36. Moore KJ, Sheedy FJ, Fisher EA. Macrophages in atherosclerosis: a dynamic balance. Nat Rev Immunol. 2013;13(10):709-721.1.

37. Van Vré EA, Van Brussel I, Bosmans JM, Vrints CJ, Bult H. Dendritic cells in human atherosclerosis: from circulation to atherosclerotic plaques. Mediators Inflamm. 2011;2011:941396.

38. Davison LM, Jorgensen TN. New treatments for systemic lupus erythematosus on the horizon: targeting plasmacytoid dendritic cells to inhibit cytokine production. J Clin Cell Immunol. 2017;8(6):534.

39. Mailer RKW, Gisterå A, Polyzos KA, Ketelhuth DFJ, Hansson GK. Hypercholesterolemia induces differentiation of regulatory T cells in the liver. Circ Res. 2017;120(11):1740-1753.

40. Florek M, et al. Loss of CD62L expression in CD4+FoxP3+regulatory T cells following freeze-and-thaw prevents protection against GVHD in murine models. Blood. 2014;124(21):2427.

41. Newton R, Priyadharshini B, Turka LA. Immunometabolism of regulatory T cells. Nat Immunol. 2016;17(6):618-625.

42. Vengrenyuk Y, et al. Cholesterol loading reprograms the microRNA-143/145-myocardin axis to convert aortic smooth muscle cells to a dysfunctional macrophage-like phenotype. Arterioscler Thromb Vasc Biol. 2015;35(3):535-546.

43. Wang F, Beck-García K, Zorzin C, Schamel WW, Davis MM. Inhibition of T cell receptor signaling by cholesterol sulfate, a naturally occurring derivative of membrane cholesterol. Nat Immunol. 2016;17(7):844-850.

44. Nurieva RI, et al. Generation of T follicular helper cells is mediated by interleukin-21 but independent of $\mathrm{T}$ helper 1,2 , or 17 cell lineages. Immunity. 2008;29(1):138-149.

45. Aprahamian T, et al. Impaired clearance of apoptotic cells promotes synergy between atherogenesis and autoimmune disease. J Exp Med. 2004;199(8):1121-1131.

46. Morel L. Mapping lupus susceptibility genes in the NZM2410 mouse model. Adv Immunol. 2012;115:113-139.

47. Ravishankar B, et al. Marginal zone CD169+ macrophages coordinate apoptotic cell-driven cellular recruitment and tolerance. Proc Natl Acad Sci U S A. 2014;111(11):4215-4220.

48. Vinuesa CG, Linterman MA, Yu D, MacLennan IC. Follicular helper T cells. Annu Rev Immunol. 2016;34:335-368

49. Choi JY, et al. Circulating follicular helper-like T cells in systemic lupus erythematosus: association with disease activity. Arthritis Rheumatol. 2015;67(4):988-999.

50. Gaddis DE, et al. Apolipoprotein AI prevents regulatory to follicular helper T cell switching during atherosclerosis. Nat Commun. 2018;9(1):1095.

51. Maganto-García E, Tarrio ML, Grabie N, Bu DX, Lichtman AH. Dynamic changes in regulatory T cells are linked to levels of diet-induced hypercholesterolemia. Circulation. 2011;124(2):185-195.

52. Mor A, Luboshits G, Planer D, Keren G, George J. Altered status of CD4(+)CD25(+) regulatory T cells in patients with acute coronary syndromes. Eur Heart J. 2006;27(21):2530-2537.

53. Lin J, Li M, Wang Z, He S, Ma X, Li D. The role of CD4+CD25+ regulatory T cells in macrophage-derived foam-cell formation. J Lipid Res. 2010;51(5):1208-1217.

54. Cheng HY, et al. Loss of ABCG1 influences regulatory T cell differentiation and atherosclerosis. J Clin Invest. 2016;126(9):3236-3246.

55. Kuznetsova T, Prange KHM, Glass CK, de Winther MPJ. Transcriptional and epigenetic regulation of macrophages in atherosclerosis. Nat Rev Cardiol. 2020;17(4):216-228.

56. Morel L, Yu Y, Blenman KR, Caldwell RA, Wakeland EK. Production of congenic mouse strains carrying genomic intervals containing SLE-susceptibility genes derived from the SLE-prone NZM2410 strain. Mamm Genome. 1996;7(5):335-339.

57. Choi SC, et al. Relative contributions of B cells and dendritic cells from lupus-prone mice to CD4 ${ }^{+} \mathrm{T}$ cell polarization. $J$ Immunol. 2018;200(9):3087-3099.

58. Yin Y, et al. Normalization of CD4+ T cell metabolism reverses lupus. Sci Transl Med. 2015;7(274):274ra18.

59. Hasham MG, et al. Systemic autoimmunity induced by the TLR7/8 agonist Resiquimod causes myocarditis and dilated cardiomyopathy in a new mouse model of autoimmune heart disease. Dis Model Mech. 2017;10(3):259-270.

60. Akkerman A, et al. CTLA4Ig prevents initiation but not evolution of anti-phospholipid syndrome in NZW/BXSB mice. Autoimmunity. 2004;37(6-7):445-451.

61. Mohan C, Alas E, Morel L, Yang P, Wakeland EK. Genetic dissection of SLE pathogenesis. Sle1 on murine chromosome 1 leads to a selective loss of tolerance to H2A/H2B/DNA subnucleosomes. J Clin Invest. 1998;101(6):1362-1372.

62. Marvin J, Rhoads JP, Major AS. Fc $\gamma$ RIIb on CD11c cells modulates serum cholesterol and triglyceride levels and differentially affects atherosclerosis in male and female $\mathrm{Ldlr}^{/ /}$mice. Atherosclerosis. 2019;285:108-119.

63. Laroumanie F, Dale BL, Saleh MA, Madhur MS. Intracellular staining and flow cytometry to identify lymphocyte subsets within murine aorta, kidney and lymph nodes in a model of hypertension. J Vis Exp. 2017;(119):55266.

64. Li W, et al. Targeting $\mathrm{T}$ cell activation and lupus autoimmune phenotypes by inhibiting glucose transporters. Front Immunol. 2019;10:833.

65. Zeng $\mathrm{H}$, et al. mTORC1 and mTORC2 kinase signaling and glucose metabolism drive follicular helper T cell differentiation Immunity. 2016;45(3):540-554 